\title{
Revising the Maximal Contrast Dose for Predicting Acute Kidney Injury following Coronary Intervention
}

\author{
Laith Hattar $^{\mathrm{a}}$ Jean-Pierre Assaker ${ }^{\mathrm{a}}$ Joe Aoun ${ }^{\mathrm{a}, \mathrm{b}}$ Lori Lyn Price ${ }^{\mathrm{c}, \mathrm{d}}$ \\ Joseph Carrozza ${ }^{a, e}$ Bertrand L. Jaber ${ }^{a, f}$ \\ aDepartment of Medicine, St. Elizabeth's Medical Center and Tufts University School of Medicine, Boston, MA, USA; \\ bDepartment of Cardiology, DeBakey Heart \& Vascular Center, Houston Methodist Hospital, Houston, TX, USA; \\ Institute for Clinical Research and Health Policy Studies, Tufts Medical Center, Boston, MA, USA; dTufts Clinical \\ and Translational Science Institute, Tufts University, Boston, MA, USA; 'Division of Cardiovascular Medicine, St. \\ Elizabeth's Medical Center, Boston, MA, USA; fDivision of Nephrology, St. Elizabeth's Medical Center, Boston, MA, \\ USA
}

\begin{abstract}
Keywords
Contrast-induced acute kidney injury · Maximal allowable contrast dose $\cdot$ Coronary angiography $\cdot$ Percutaneous coronary intervention $\cdot$ Youden index $\cdot$ Risk prediction
\end{abstract}

\begin{abstract}
Introduction: The maximal allowable contrast dose (MACD $=5 \times$ body weight $/$ serum creatinine) is an empiric equation that has been used and validated in several studies to mitigate the risk of contrast-induced acute kidney injury (CI-AKI). However, coefficient 5 (referred to as factor $K$ ) was empirically devised and never disputed. The aim of this study was to refine the MACD equation for the prediction of $\mathrm{Cl}-\mathrm{AKI}$ following percutaneous coronary interventions ( $\mathrm{PCls})$. Methods: This is a single-center, retrospective cohort study of adults undergoing $\mathrm{PCl}$. Electronic medical records were reviewed to identify patients who underwent $\mathrm{PCl}$ between 2010 and 2019, derived from the National Cardiovascular Data Registry Cath-PCl registry for our hospital. Factor K (de-
\end{abstract}

fined as contrast volume $\times$ serum creatinine/body weight) was calculated for every patient. A receiver operating characteristic (ROC) curve was constructed, and the Youden index was used to identify the optimal cut-off value for factor $K$ in predicting severe (stages 2-3) Cl-AKI. Results: Of the 3,506 patients undergoing $\mathrm{PCl}, 255$ (7.2\%) developed Cl-AKI, and $68(26.7 \%)$ of the 255 experienced severe AKI. Factor $K$ predicted all-stage $\mathrm{Cl}$-AKI (area under the ROC curve 0.649; $95 \% \mathrm{Cl} 0.611,0.686)$ but had better performance for predicting severe (stages $2-3)$ AKI $(0.736 ; 95 \% \mathrm{Cl} 0.674,0.800)$. The optimal cut-off value for factor $K$ in predicting severe $\mathrm{CI}$-AKI was 2.5 , with a corresponding sensitivity of $68.7 \%$ and specificity of $70.5 \%$. On subgroup analyses, optimal cut-off values for factor $K$ for high-risk groups were not significantly different from those of low-risk groups. Conclusion: Our study indicates that factor $K$ in the MACD equation is an independent risk factor for the development of severe $\mathrm{Cl}-\mathrm{AKI}$, with an optimal cut-off value of 2.5. If our findings are validated, the MACD equation should be revised to incorporate the coefficient of 2.5 instead of 5 . (c) 2021 S. Karger AG, Basel karger@karger.com

www.karger.com/ajn
(C) 2021 S. Karger AG, Basel 


\section{Introduction}

Contrast-induced acute kidney injury (CI-AKI) is a serious hospital-acquired condition, with an incidence rate of 3-19\% following cardiovascular procedures [1]. Once it develops, CI-AKI has short- and long-term implications, conferring an increased risk of adverse clinical outcomes, including prolonged hospital length of stay, higher in-hospital mortality [2], long-term decline in kidney function [3], and greater healthcare expenditures [4]. Once diagnosed, the clinical management of CI-AKI is largely supportive.

While nonmodifiable risk factors for CI-AKI include advanced age, diabetes mellitus, heart failure, and preexisting CKD, modifiable risk factors include extracellular fluid volume depletion and both the type and volume of contrast agent administered. Regarding the volume of contrast agent administered, no single, universally accepted threshold has been associated with the development of CI-AKI. Suggested volume cut-off values have included a fixed volume of $125 \mathrm{~mL}$ [5], a weight-based volume of $3 \mathrm{~mL} / \mathrm{kg}$ [6], and the use of the maximal allowable contrast dose (MACD), originally conceptualized by Cigarroa et al. [7]. The MACD is defined by the following empiric equation of 5 multiplied by body weight (in $\mathrm{kg}$ ) and divided by the serum creatinine (Cr) (in $\mathrm{mg} / \mathrm{dL}$ ), with a maximum dose of $300 \mathrm{~mL}$. While the authors originally hypothesized a relationship between the contrast dose or volume, the body weight, and the baseline kidney function, there was no scientific basis for selecting the coefficient of 5. Nevertheless, exceeding the MACD has been associated with an increased risk of CI-AKI [8]. Despite its use and validation in retrospective and prospective cohort studies [9-13], the origin of factor 5, thereafter termed factor " $K$," in the equation has not been disputed.

The aim of this diagnostic test study was to redefine the MACD equation among patients undergoing percutaneous coronary intervention (PCI), with a focus on refining the coefficient factor " $K$," identifying a new cut-off value to improve the prediction of all-stage and severe CI-AKI, and exploring different cut-off values for subgroups of patients at higher risk for CI-AKI.

\section{Methods}

\section{Study Design}

This was a single-center, retrospective cohort study of adults undergoing PCI at an acute care facility (St. Elizabeth's Medical Center, Boston, MA, USA) over a 10-year period (January 4, 2010January 3,2020 ) with a suspected or confirmed diagnosis of coro- nary artery disease warranting a coronary angiogram. The study was approved by the Institutional Review Board, and requirement for informed consent was waived due to the retrospective nature of the study. St. Elizabeth's Medical Center is a 273-bed acute care teaching hospital, affiliated with Tufts University School of Medicine, located in Boston, and serves as the tertiary care hospital for the Massachusetts region of the Steward Health Care System (Dallas, TX, USA), which operates 9 hospitals in eastern Massachusetts.

\section{Data Source and Study Population}

The primary source for the analysis was the clinical data repository derived from the National Cardiovascular Data Registry (NCDR) Cath-PCI registry for our hospital. The NCDR Cath-PCI registry is cosponsored by the American College of Cardiology and the Society for Cardiovascular Angiography and Interventions, and collects data on patient and hospital characteristics, clinical presentation, treatments, and outcomes associated with PCIs from hospitals across the USA [14]. At participating institutions, the data are entered into a certified software with a comprehensive data quality and audit program, and are then exported to a national data repository at the American College of Cardiology warehouse.

For our study, we identified all adults (age $\geq 18$ years) undergoing PCI between January 04, 2010, and January 3, 2020, enrolled in the NCDR Cath-PCI $(n=3,794)$. We excluded patients without a preprocedure or a peak postprocedure in-hospital serum $\mathrm{Cr}$ value $(n=257)$ and patients receiving dialysis at the time of the PCI for acute or chronic kidney failure $(n=31)$. The final analytical cohort included 3,506 patients undergoing PCI.

\section{Data Collection and Definitions of Variables}

Data collection for the cohort included demographic and clinical variables (age, gender, race/ethnicity, weight, height, BMI, and tobacco use), co-existing conditions (hypertension, coronary artery disease [prior myocardial infraction or PCI], cerebrovascular disease, peripheral vascular disease, diabetes mellitus, heart failure, and chronic lung disease), laboratory variables (pre-PCI and postPCI peak serum $\mathrm{Cr}$ ), the baseline (pre-PCI) estimated Cr clearance using the Cockcroft-Gault equation [15], the baseline (pre-PCI) estimated glomerular filtration rate (eGFR) using the CKD-Epi equation $[16,17]$, and the outcome variable of interest (development of CI-AKI). To improve the accuracy of the Cockcroft-Gault equation, we used one of the following body weights, based on the BMI: actual body weight (for BMI $<18.5 \mathrm{~kg} / \mathrm{m}^{2}$ ), ideal body weight (IBW) (for BMI 18.5-24.9 kg/m²), and adjusted body weight (ABW) (for BMI $\geq 25 \mathrm{~kg} / \mathrm{m}^{2}$ ). The IBW was estimated by the following equations: IBW $(\mathrm{kg})=50+(0.91 \times$ [height in centimeters $-152.4])$ for males and $45.5+(0.91 \times$ [height in centimeters -152.4]) for females [18]. The ABW was estimated by the following equation: $\mathrm{ABW}(\mathrm{kg})=\mathrm{IBW}(\mathrm{kg})+(0.4 \times$ [actual body weight $(\mathrm{kg})$ - IBW] $[19]$. The presence of CKD was defined based on a preprocedure eGFR of $<60 \mathrm{~mL} / \mathrm{min} / 1.73 \mathrm{~m}^{2}$.

The definition of the pre- and post-PCI serum $\mathrm{Cr}$ variable evolved over the course of the study. For the period of 2010-2016, in accordance with the NCDR predefined data specifications version 4.4, the pre-PCI serum Cr represented a value within 1 month prior to the procedure, and the post-PCI serum $\mathrm{Cr}$ represented a peak value within 30 days, until discharge, or until the next procedure. For the period of 2017-2020 and in accordance with the NCDR predefined data specifications version 5.0 , the post-PCI se- 
Table 1. Characteristics of patients with and without CI-AKI

\begin{tabular}{|c|c|c|c|}
\hline Characteristic & $\begin{array}{l}\text { Patients without } \\
\text { CI-AKI }(n=3,251)\end{array}$ & $\begin{array}{l}\text { Patients with } \\
\text { CI-AKI }(n=255)\end{array}$ & $p$ value \\
\hline Mean age, years & $66.7(12.6)$ & $72.3(12.3)$ & $<0.001$ \\
\hline Female gender & $963(29.6)$ & $87(34.1)$ & 0.13 \\
\hline \multicolumn{4}{|l|}{ Race/ethnicity } \\
\hline White & $2,892(90.5)$ & $218(86.2)$ & \\
\hline Black & $159(5.0)$ & $25(9.9)$ & 0.004 \\
\hline Other & $145(4.5)$ & $10(3.9)$ & \\
\hline BMI, $\mathrm{kg} / \mathrm{m}^{2}$ & $29.6(6.1)$ & $29.3(6.2)$ & 0.43 \\
\hline \multicolumn{4}{|l|}{ Pre-existing conditions } \\
\hline Hypertension & $2,841(87.4)$ & $234(91.8)$ & 0.04 \\
\hline Hyperlipidemia & $2,819(86.7)$ & $220(86.3)$ & 0.84 \\
\hline Cardiovascular disease & $446(13.7)$ & $44(17.3)$ & 0.12 \\
\hline Diabetes mellitus & $1,280(39.4)$ & $132(51.8)$ & $<0.001$ \\
\hline Heart failure & $859(26.4)$ & $156(61.2)$ & $<0.001$ \\
\hline Chronic lung disease & $746(23.0)$ & $78(30.6)$ & 0.006 \\
\hline Peripheral arterial disease & $698(21.5)$ & $91(35.7)$ & $<0.001$ \\
\hline $\mathrm{CKD}$ & $902(28.2)$ & $160(63.2)$ & $<0.001$ \\
\hline \multicolumn{4}{|l|}{ Serum Cr, mg/dL } \\
\hline Preprocedure & $1.0(0.4)$ & $1.4(0.7)$ & $<0.001$ \\
\hline Postprocedure & $1.0(0.3)$ & $2.4(1.4)$ & $<0.001$ \\
\hline Baseline Cr clearance, $\mathrm{mL} / \mathrm{min}$ & $76.0(32.3)$ & $55.8(30.7)$ & $<0.001$ \\
\hline Baseline eGFR, $\mathrm{mL} / \mathrm{min} / 1.73 \mathrm{~m}^{2}$ & $74.0(22.4)$ & $56.0(24.0)$ & $<0.001$ \\
\hline
\end{tabular}

Continuous variables are displayed as means (with standard deviation), and binary variables as counts (with percentages). CI-AKI, contrast-induced acute kidney injury; eGFR, estimated glomerular filtration rate.

rum $\mathrm{Cr}$ represented the highest value 5 days after the procedure, at discharge, or until the next procedure. Factor $K$ was calculated for every patient using the following equation: $K=$ contrast volume $(\mathrm{mL}) \times$ serum $\mathrm{Cr}[\mathrm{mg} / \mathrm{dL}] /$ body weight $[\mathrm{kg}])$.

\section{Predictor and Outcome Variables}

The main predictor variable of interest was factor " $K$ " of the MACD equation. The outcome variable of interest was CI-AKI, based on the definition and staging system proposed by the Kidney Disease Improving Global Outcomes (KDIGO) clinical practice guideline for AKI [20], using the pre- to post-PCI (peak) serum $\mathrm{Cr}$ change. In brief, CI-AKI was defined by an absolute increase of 0.3 $\mathrm{mg} / \mathrm{dL}$ or greater or a relative 1.5 -fold increase or greater in serum $\mathrm{Cr}$ pre- to postprocedure. The following stages of AKI were assessed: stage 1 (increase in serum $\mathrm{Cr}$ by $\geq 0.3 \mathrm{mg} / \mathrm{dL}$ or 1.5 - to 2 -fold increase in serum $\mathrm{Cr}$ ); stage 2 ( $>2$ - to 3 -fold increase in serum $\mathrm{Cr}$ ); and stage 3 ( $>3$-fold increase in serum $\mathrm{Cr}$, or serum $\mathrm{Cr}$ $\geq 4.0 \mathrm{mg} / \mathrm{dL}$ with an acute increase of $>0.5 \mathrm{mg} / \mathrm{dL}$ ). Dialysis-requiring CI-AKI was an NCDR predefined in-hospital outcome, consisting of worsening kidney failure necessitating new dialysis. Mild CI-AKI was defined as stage $1 \mathrm{AKI}$, and severe CI-AKI was defined as stages 2-3 AKI or dialysis requirement.

\section{Statistical Analyses}

Continuous variables are reported as means (with standard deviation). Binary variables are reported as counts (with percentages). Comparisons between patients with and without CI-AKI were made by the Student's $t$ test for continuous variables and by the $\chi^{2}$ test for categorical variables.

Receiver operating characteristic (ROC) curve analyses were performed to test the performance characteristic of the variable of interest, factor $K$, for prediction of CI-AKI (stages 1-3) and severe CI-AKI (stages 2-3). The 95\% CI for the area under the ROC curve was calculated, and formal statistical testing was performed using the method described by Gonen [21]. The optimal cut-off point for the development of CI-AKI was determined for factor $K$ using the Youden index [22], based on which sensitivity, specificity, and positive and negative predictive values were calculated. The Youden index is calculated by adding sensitivity and specificity and subtracting 1, which identifies the point on the ROC curve that has the maximum vertical distance to the diagonal or chance line. Analyses were performed to identify the optimal factor $K$ cut-off point for the development of severe CI-AKI in the entire cohort and in the following prespecified high-risk groups: age ( $<65$ vs. $\geq$ 65 years), diabetes mellitus (present vs. absent), heart failure (present vs. absent), and CKD (present vs. absent).

A separate ROC curve analysis was performed to test the performance characteristic of the contrast volume-to-eGFR ratio for the prediction of severe CI-AKI. Logistic regression analysis was also used to examine the predictive value of factor $K$ and contrast volume-to-Cr clearance for the development of CI-AKI. Adjustment variables included age, gender, race, baseline eGFR, diabetes mellitus, and heart failure. The results of the logistic regression analyses are displayed as odds ratios with $95 \%$ CI. 


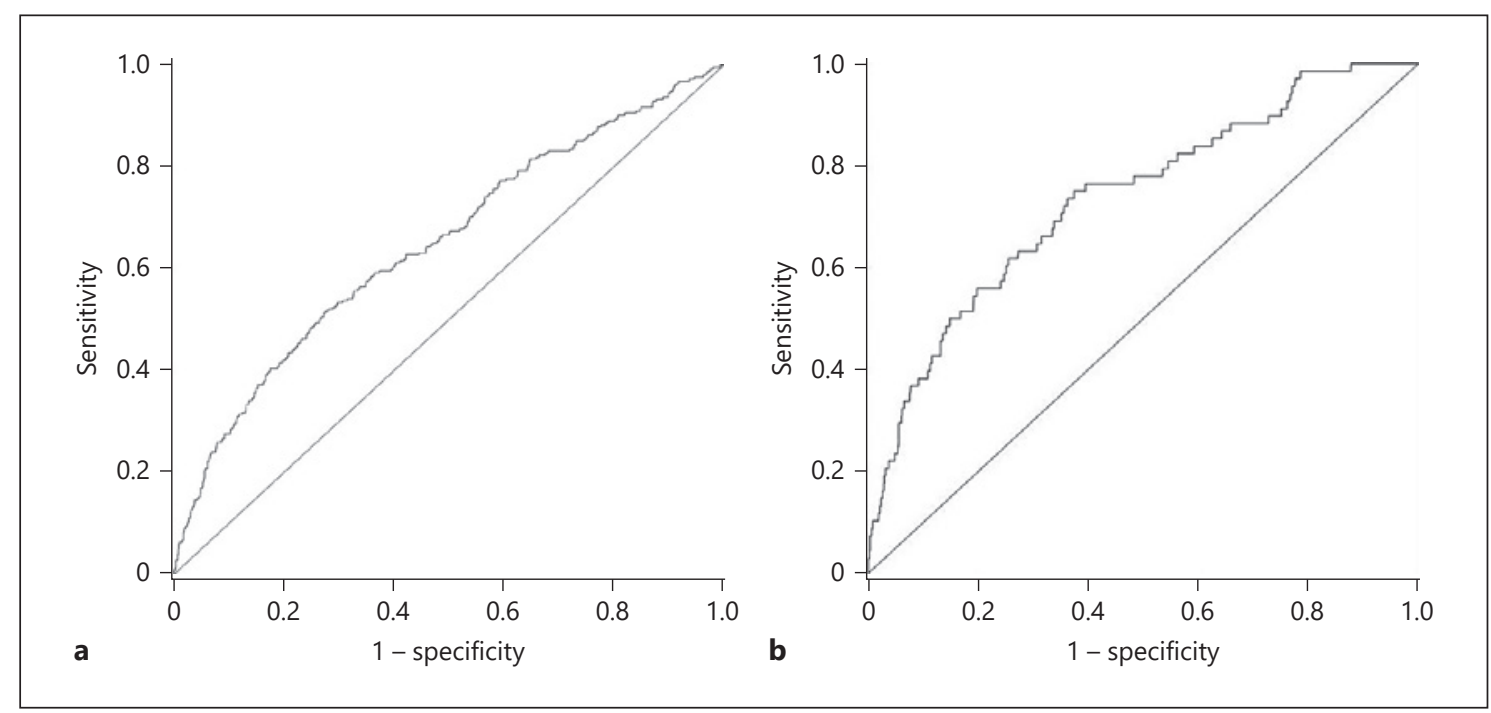

Fig. 1. ROC curve displaying the performance of factor " $K$ " for the prediction of (a) mild to severe (stages 1-3) and (b) severe (stages 2-3) CI-AKI following PCI. The area under the ROC curve is $0.649(95 \%$ CI $0.611,0.686)$ for the prediction of mild to severe CI-AKI, and 0.736 (95\% CI 0.674, 0.800) for the prediction of severe CI-AKI. ROC, receiver operating characteristic; CI-AKI, contrast-induced acute kidney injury; PCI, percutaneous coronary intervention.

The functional form of factor $K$ and its association with the incidence rate of CI-AKI was also examined using a restricted cubic spline with 4 knots. The plot of the restricted spline was constructed using the design library of R version 2.6 (Free Software Foundation, Boston, MA, USA). The pROC package in $\mathrm{R}$ was used to determine the optimal cut-off point values based on the Youden Index [23]. All other analyses and plots were generated using SAS version 9.4 (SAS Institute Inc., Cary, NC, USA). Differences were considered statistically significant at a $p$ value of $<0.05$.

\section{Results}

Characteristics of Patients with and without CI-AKI

Following PCI, 255 (7.2\%) patients developed CI-AKI, with 68 (26.7\%) of the 255 patients experiencing severe (stages 2-3) AKI. Table 1 summarizes the characteristics of the 3,506 patients undergoing PCI according to the development of CI-AKI status. In brief, patients with CIAKI were older (72 vs. 67 years; $p<0.001$ ) and had a higher prevalence of hypertension ( 91.8 vs. $87.4 \%$; $p=$ 0.04 ), diabetes mellitus (51.8 vs. $39.4 \%$; $p<0.001$ ), heart failure (61.2 vs. $26.4 \% ; p<0.001)$, chronic lung disease (30.6 vs. $23.0 \% ; p=0.006$ ), peripheral arterial disease (35.7 vs. $21.5 \%$; $p<0.001$ ), and CKD (63.2 vs. $28.2 \%$; $p<$ $0.001)$, than patients who did not develop CI-AKI.

The total contrast volume administered was not significantly different between patients with and without
CI-AKI $(202.0 \pm 82.3 \mathrm{~mL}$ vs. $196.0 \pm 72.9 \mathrm{~mL} ; p=0.21)$. However, the contrast volume-to-Cr clearance ratio was significantly higher among patients with CI-AKI (4.5 \pm 2.8 vs. $3.1 \pm 1.8 ; p<0.001)$.

\section{ROC Curve Analyses for the Diagnostic Performance of Factor $K$}

The results of the ROC curve analyses for the diagnostic performance of factor $K$ for the development of CIAKI, including severe (stages 2 and 3) AKI, are summarized in Figure 1 . In brief, factor $K$ modestly predicted the development of CI-AKI (stages 1-3) with an area under the ROC curve of 0.649 (95\% CI 0.611, 0.686; Fig. 1a) but had better performance for the prediction of severe (stages 2-3) AKI with an area under the ROC curve of 0.736 (95\% CI 0.674, 0.800; Fig. 1b). We next focused on refining the performance of factor $K$ for predicting severe CIAKI due to its associated morbidity. In a separate ROC curve analysis, the contrast volume-to-eGFR ratio also displayed good diagnostic performance for predicting severe (stages 2-3) AKI with an area under the ROC curve of 0.779 (95\% CI $0.723,0.835$ ).

\section{Optimal Cut-Off Value for Factor K for Prediction of} Severe (Stages 2-3) CI-AKI

To identify a potential new cut-off value for factor $K$, set empirically at " 5 " in the originally described MACD 
Table 2. Optimal factor $K$ cut-off value for prediction of severe (stages 2 and 3) CI-AKI (derived from the Youden index)

\begin{tabular}{|c|c|c|c|c|c|c|c|}
\hline & $\begin{array}{l}\text { Optimal factor } \\
K \text { cut-off value* }\end{array}$ & Sensitivity & Specificity & $\begin{array}{l}\text { Negative } \\
\text { predictive value }\end{array}$ & $\begin{array}{l}\text { Positive } \\
\text { predictive value }\end{array}$ & $\begin{array}{l}\text { Area under the } \\
\text { ROC curve }(95 \% \mathrm{CI})\end{array}$ & $p$ value ${ }^{* *}$ \\
\hline All patients & 2.5 & $75.0 \%$ & $62.6 \%$ & $99.2 \%$ & $4.0 \%$ & $0.74(0.67,0.80)$ & - \\
\hline \multicolumn{8}{|l|}{ Age, years $(n)$} \\
\hline$<65(1,507)$ & 2.5 & $78.9 \%$ & $74.2 \%$ & $99.6 \%$ & $3.9 \%$ & $0.81(0.71,0.91)$ & \multirow{2}{*}{0.05} \\
\hline$\geq 65(1,999)$ & 3.2 & $57.1 \%$ & $74.6 \%$ & $98.5 \%$ & $5.7 \%$ & $0.68(0.60,0.77)$ & \\
\hline \multicolumn{8}{|l|}{ Diabetes mellitus $(n)$} \\
\hline Absent $(2,094)$ & 2.6 & $80.8 \%$ & $63.8 \%$ & $99.6 \%$ & $2.9 \%$ & $0.76(0.67,0.84)$ & \multirow{2}{*}{0.68} \\
\hline Present $(1,412)$ & 2.9 & $64.3 \%$ & $73.4 \%$ & $98.4 \%$ & $7.3 \%$ & $0.73(0.64,0.81)$ & \\
\hline \multicolumn{8}{|l|}{ Heart failure $(n)$} \\
\hline Absent $(2,491)$ & 2.5 & $77.3 \%$ & $65.0 \%$ & $99.7 \%$ & $2.0 \%$ & $0.73(0.61,0.85)$ & \multirow{2}{*}{0.74} \\
\hline Present $(1,015)$ & 2.9 & $67.4 \%$ & $68.1 \%$ & $97.5 \%$ & $10.2 \%$ & $0.70(0.62,0.78)$ & \\
\hline \multicolumn{8}{|l|}{$\operatorname{CKD}(n)$} \\
\hline Absent $(1,119)$ & 1.5 & $94.7 \%$ & $28.9 \%$ & $99.8 \%$ & $1.1 \%$ & $0.62(0.49,0.74)$ & \multirow[b]{2}{*}{0.49} \\
\hline Present $(2,387)$ & 3.6 & $62.5 \%$ & $67.2 \%$ & $97.1 \%$ & $9.2 \%$ & $0.67(0.59,0.75)$ & \\
\hline
\end{tabular}

ROC, receiver operating characteristic; CI-AKI, contrast-induced acute kidney injury. ${ }^{*}$ Derived from the Youden index. ${ }^{* *} p$ value for comparison of area under the curve within groups.

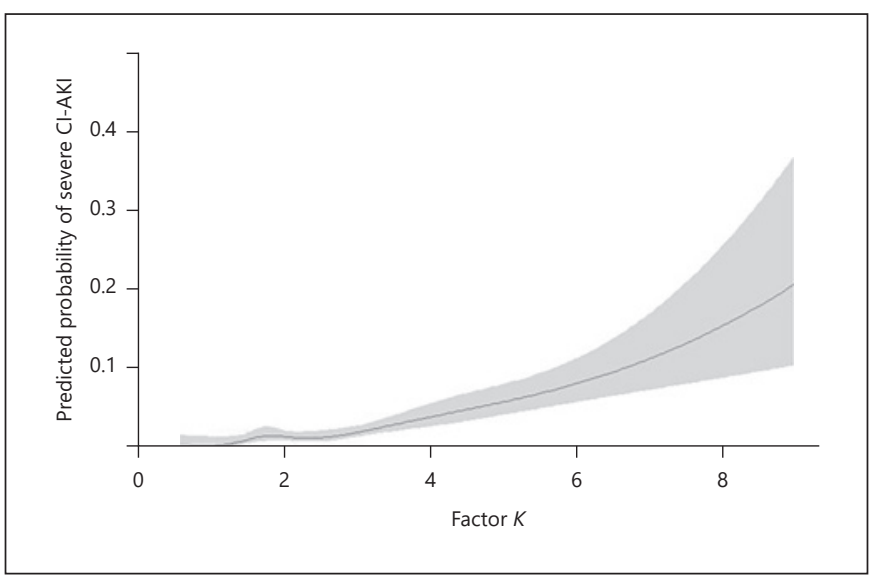

Fig. 2. Restrictive cubic spline depicting the unadjusted relationship between factor " $K$ " and incidence of severe (stages 2-3) CIAKI following PCI. Shaded area represents the 95\% CI. CI-AKI, contrast-induced acute kidney injury; PCI, percutaneous coronary intervention.

equation, we first constructed a restrictive cubic spline to ascertain the crude relationship between factor $K$ and severe CI-AKI. As shown in Figure 2, an almost linear relationship was observed.

Using the Youden index, we next explored the optimal factor $K$ cut-off value and the corresponding sensitivity, specificity, and positive and negative predictive values for the prediction of severe CI-AKI in the entire cohort and in the high-risk groups of interest. As shown in Table 2, at a cut-off value of 2.5 , factor $K$ displayed the most optimal sensitivity and specificity of $75.0 \%$ and $62.6 \%$, respectively. In a sensitivity analysis where severe CI-AKI was defined by an absolute increase in serum $\mathrm{Cr}$ of $1.0 \mathrm{mg} / \mathrm{dL}$ or greater, the optimal factor $K$ cut-off value was 2.8 , with a corresponding sensitivity and specificity of $68.3 \%$ and $71.5 \%$, respectively. In subgroup analyses, we found no differential optimal cut-off value for factor $K$ according to age or the presence of diabetes mellitus, heart failure, or CKD.

\section{Logistic Regression Analyses}

In a univariate analysis, factor $K$ was associated with higher odds for the development of severe (stages 2-3) CI-AKI (unadjusted odds ratio 1.67; 95\% CI 1.48, 1.88; $p<0.001)$. After adjustment for known confounders of this association, including age, sex, race, diabetes mellitus, heart failure, and baseline eGFR, factor $K$ remained independently associated with higher odds for the development of severe CI-AKI (adjusted odds ratio 1.29; 95\% CI $1.10,1.51 ; p=0.002)$.

\section{Discussion}

Cigarroa et al. [7] first coined the MACD, which was derived from the empiric equation for dosing of contrast agents in patients with kidney disease undergoing cardiovascular procedures. The equation incorporated the pa- 
tient's body weight (in $\mathrm{kg}$ ) and serum $\mathrm{Cr}$ value (in $\mathrm{mg}$ / $\mathrm{dL}$ ), and basically called for a maximum contrast agent administration volume of $5 \mathrm{~mL} / \mathrm{kg}$ of body weight, index to the serum Cr value. The study found that patients who exceeded the maximum dose allowed had a significantly higher risk of CI-AKI [7]. While there has been no scientific basis for selecting the coefficient of 5 for the MACD equation (referred to as factor $K$ ), several studies have subsequently validated the predictive value of this equation [9-12, 24-26]. It should be noted that the MACD equation was empirically developed based on knowledge that more contrast volume can be injurious to the kidneys and that lower GFR limits the volume of contrast that can be administered, rather than being derived from a formal statistical evaluation of the outcome of CI-AKI. Our study aimed to revise the equation and to optimize the factor $K$ value to achieve the highest sensitivity and specificity to detect severe (stages 2 and 3) CI-AKI.

In the present diagnostic test study, using a large, unselected cohort of patients undergoing PCI at an acute care facility, over a 10-year period, we observed a relatively low incidence rate of CI-AKI of 7.2\%. We found an almost linear relationship between factor $K$ and the incidence rate of severe (stages 2 and 3) CI-AKI. We next explored the optimal factor $K$ cut-off value for the prediction of severe CI$\mathrm{AKI}$ in the entire cohort and in high-risk groups, and found that in the entire cohort, a cut-off value of 2.5 (rather than the empirical value of 5) displayed the best sensitivity and specificity. We were unable to identify a differential cut-off point value for factor $K$ according to prespecified high-risk groups. Our study results are consistent with previous literature, suggesting that a higher contrast dose is associated with an increased risk of CI-AKI [6, 27-29]. After accounting for non-modifiable risk markers that can confound our results, including age, sex, race, and the presence of comorbidities including diabetes mellitus, CKD, and heart failure, factor $K$ remained an independent risk factor for the development of CI-AKI. Our study also found that factor $K$ was a better predictor of severe CI-AKI rather than all-stage CIAKI. Our analysis suggests that a lower factor $K$ value, half of the original numerical value (i.e., 2.5 instead of 5), provided better performance characteristics for the detection of severe CI-AKI.

In the original report describing the MACD, patients undergoing coronary angiography received diatrizoate (Renografin-76), a hyperosmolar contrast agent that is potentially more injurious to the kidneys and no longer in use, and AKI was defined as a rise in serum $\mathrm{Cr}$ of $1 \mathrm{mg} / \mathrm{dL}$ or greater [7]. High-osmolar contrast agents have since been replaced by low-osmolar (e.g., iohexol) and iso-osmolar

Maximal Allowable Contrast Dose and Acute Kidney Injury (e.g., iodixanol) agents. In our study, patients received iohexol, consistent with current clinical practice. Furthermore, the definition of AKI has evolved over time, and we used the currently accepted consensus definition. In a sensitivity analysis, using the AKI definition from the original report, the optimal factor $K$ cut-off value was 2.8 (instead of 2.5). This is not surprising given that a higher volume of contrast would be required to cause more severe AKI. The contrast volume-to-eGFR ratio, a parameter that does not factor in body weight, was originally proposed by Raposeiras-Roubin et al. to stratify the risk of CI-AKI in patients undergoing coronary angiography [12]. In our study, this ratio was shown to predict severe AKI better than factor $K$.

Our study has several strengths. We analyzed a large, diverse, and unselected adult cohort of patients undergoing PCI. Our main finding, if externally validated, is of substantial importance, as measures to further limit exposure to higher doses of contrast can help further decrease the incidence of CI-AKI, a hospital-acquired condition that is increasingly being recognized as a patient safety indicator [30,31]. Our analysis calls for the need to refine the MACD equation to replace the 5.0 coefficient factor with 2.5 (or factor $K$ ). The revised MACD equation should be incorporated into quality improvement initiatives aimed at standardizing practice pattern variation in contemporary PCI with the ultimate lofty goal of eliminating CI-AKI. Previous efforts incorporating a multipronged quality improvement intervention, including the MACD, have clearly demonstrated a reduction in rates of CI-AKI following PCI [32].

There are also several important limitations to consider. The study was retrospective in nature and was conducted at a single acute-care facility, limiting the generalizability of the findings. Our definition of CI-AKI may have underestimated the true incidence and stages of severity as we relied on the pre- to post-PCI serum $\mathrm{Cr}$ change, derived from the NCDR predefined data specifications, which evolved during the course of our study, including a change to the peak serum Cr time frame. Indeed, for the study period of 2010-2016, the post-PCI serum $\mathrm{Cr}$ represented a peak value within 30 days, until discharge, or until the next procedure, whereas for the study period of 2017-2019, the post-PCI serum Cr represented the highest value within 5 days after the procedure, at discharge, or until the next procedure. As a result, the potential for misclassification cannot be ruled out. To help overcome this challenge, we limited our main outcome variable of interest to severe AKI, as defined by stages 2 and 3, which is less likely to be misclassified based on a larger serum $\mathrm{Cr}$ increment or requirement for dialysis.

Am J Nephrol 2021;52:328-335 
We observed a relatively lower incidence rate of CI-AKI at our facility. Of note, a number of patients $(\sim 14 \%$ of the parent cohort) were excluded from the study due to early discharge with the absence of pre- and/or post-PCI serum $\mathrm{Cr}$ value, which might have contributed to an ascertainment bias, resulting in lower AKI rates. Nevertheless, the observed lower AKI rate likely represents widespread adoption of standardized hydration protocols for patients with pre-PCI eGFR $<60 \mathrm{~mL} / \mathrm{min} / 1.73 \mathrm{~m}^{2}$, use of the transradial approach, use of small catheters to limit contrast volume, use of low-osmolar contrast agents, avoidance of test injections and ventriculography, and, when indicated, staging an intervention. Our subgroup analyses revealed, paradoxically, a slightly higher optimal cut-off value for factor $\mathrm{K}$ in the high-risk groups, including older patients ( $\geq 65$ years) and patients with diabetes, CKD, and heart failure. However, the $K$ value was not significantly different between the low- and high-risk groups. We can only speculate as to whether the higher $K$ values might reflect a more focused application of the aforementioned preventative measures to these higher risk groups of patients compared to the lower risk population.

In conclusion, this study provides support to the hypothesis that the MACD equation, a previously validated tool aimed at limiting contrast volume exposure during cardiovascular procedures, would benefit from a revision. Our study suggests that a lower factor $K$ (cut-off value of 2.5 instead of 5) is a better predictor of severe CI-AKI. If our findings are externally validated, the MACD equation should be revised as follows: 2.5 (instead of 5) multiplied by body weight (in $\mathrm{kg}$ ) and divided by serum $\mathrm{Cr}$ (in $\mathrm{mg} / \mathrm{dL}$ ).

\section{Acknowledgements}

This work was presented in part at the Annual Research Day of St. Elizabeth's Medical Center, Boston, MA, USA (March 28, 2019), and the American College of Cardiology Annual Meeting, San Antonio, TX, USA (March 29, 2020).

\section{Statement of Ethics}

The study was approved by the Institutional Review Board (No. HW200), and requirement for informed consent was waived due to the retrospective nature of the study.

\section{Conflict of Interest Statement}

The authors declare that they have no relevant financial interests pertaining to this study.

\section{Funding Sources}

This project was supported in part by the National Center for Research Resources Grant Number UL1 RR025752 and the National Center for Advancing Translational Sciences, National Institutes of Health, Grant Numbers UL1 TR000073 and UL1 TR001064. The content is solely the responsibility of the authors and does not necessarily represent the official views of the National Center for Research Resources or the National Institutes of Health.

\section{Author Contributions}

Research idea and study design: J.A. and B.L.J.; data acquisition: J.A., J.P.A., and L.H.; data analysis/interpretation: L.L.P., L.H., J.P.C., and B.L.J.; statistical analysis: L.L.P.; supervision or mentorship: B.L.J. Each author contributed important intellectual content during manuscript drafting or revision and accepts accountability for the overall work by ensuring that questions pertaining to the accuracy or integrity of any portion of the work are appropriately investigated and resolved. B.L.J. takes responsibility that this study has been reported honestly, accurately, and transparently; that no important aspects of the study have been omitted; and that any discrepancies from the study as planned have been explained.

\section{References}

1 Weisbord SD, Mor MK, Resnick AL, Hartwig KC, Sonel AF, Fine MJ, et al. Prevention, incidence, and outcomes of contrast-induced acute kidney injury. Arch Intern Med. 2008; 168(12):1325-32

2 Weisbord SD, Palevsky PM. Contrast-induced acute kidney injury: short- and longterm implications. Semin Nephrol. 2011; 31(3):300-9.
3 James MT, Ghali WA, Tonelli M, Faris P, Knudtson ML, Pannu N, et al. Acute kidney injury following coronary angiography is associated with a long-term decline in kidney function. Kidney Int. 2010;78(8):803-9.

4 Subramanian S, Tumlin J, Bapat B, Zyczynski T. Economic burden of contrast-induced nephropathy: implications for prevention strategies. J Med Econ. 2007;10(2):119-34.
5 Rich MW, Crecelius CA. Incidence, risk factors, and clinical course of acute renal insufficiency after cardiac catheterization in patients 70 years of age or older: a prospective study. Arch Intern Med. 1990;150:1237-42.

6 Moscucci M. Grossman \& Baim's cardiac catheterization, angiography, and intervention. 8th ed. Philadelphia, PA: Lippincott Williams \& Wilkins; 2014. 
7 Cigarroa RG, Lange RA, Williams RH, Hillis LD. Dosing of contrast material to prevent contrast nephropathy in patients with renal disease. Am J Med. 1989;86(6 Pt 1):649-52.

8 Aoun J, Nicolas D, Brown JR, Jaber BL. Maximum allowable contrast dose and prevention of acute kidney injury following cardiovascular procedures. Curr Opin Nephrol Hypertens. 2018;27(2):121-9.

9 Freeman RV, O'Donnell M, Share D, Meengs WL, Kline-Rogers E, Clark VL, et al. Blue cross-blue shield of michigan cardiovascular consortium (BMC2): nephropathy requiring dialysis after percutaneous coronary intervention and the critical role of an adjusted contrast dose. Am J Cardiol. 2002;90:106873.

10 Marenzi G, Assanelli E, Campodonico J, Lauri G, Marana I, De Metrio M, et al. Contrast volume during primary percutaneous coronary intervention and subsequent contrastinduced nephropathy and mortality. Ann Intern Med. 2009;150(3):170-7.

11 Brown JR, Robb JF, Block CA, Schoolwerth AC, Kaplan AV, O'Connor GT, et al. Does safe dosing of iodinated contrast prevent contrast-induced acute kidney injury? Circ Cardiovasc Interv. 2010;3(4):346-50.

12 Raposeiras-Roubín S, Abu-Assi E, OcaranzaSánchez R, Alvarez-Álvarez B, CambeiroGonzález C, Fandiño-Vaquero R, et al. Dosing of iodinated contrast volume: a new simple algorithm to stratify the risk of contrast-induced nephropathy in patients with acute coronary syndrome. Catheter Cardiovasc Interv. 2013;82(6):888-97.

13 Mauro M, Rogers EK, Cecelia M, Smith Dean E, David S, O'Donnell M, et al. Association of a continuous quality improvement initiative with practice and outcome variations of contemporary percutaneous coronary interventions. Circulation. 2006;113:814-22.

14 Brindis RG, Fitzgerald S, Anderson HV, Shaw RE, Weintraub WS, Williams JF. The American college of cardiology-national cardiovascular data registry (ACC-NCDR): building a national clinical data repository. J Am Coll Cardiol. 2001;37(8):2240-5.
15 Cockcroft DW, Gault MH. Prediction of creatinine clearance from serum creatinine. Nephron. 1976;16(1):31.

16 Levey AS, Stevens LA, Schmid CH, Zhang YL, Castro AF, Feldman HI, et al. CKD-EPI (chronic kidney disease epidemiology collaboration): a new equation to estimate glomerular filtration rate. Ann Intern Med. 2009;150: 604-12.

17 Inker LA, Schmid CH, Tighiouart H, Eckfeldt JH, Feldman HI, Greene T, et al. CKD-EPI investigators: estimating glomerular filtration rate from serum creatinine and cystatin C. N Engl J Med. 2012;367(1):20-9.

18 Winter MA, Guhr KN, Berg GM. Impact of various body weights and serum creatinine concentrations on the bias and accuracy of the cockcroft-gault equation. Pharmacotherapy. 2012;32(7):604-12.

19 Erstad BL. Dosing of medications in morbidly obese patients in the intensive care unit setting. Intensive Care Med. 2004;30(1):18-32.

20 Kellum JA, Lameire N, Aspelin P, Barsoum RS, Burdmann EA, Goldstein SL, et al. Kidney disease: improving global outcomes (KDIGO) acute kidney injury work group. KDIGO clinical practice guideline for acute kidney injury. Kidney Int Suppl. 2012;2(1):138.

21 Gonen M. Analyzing receiver operating characteristic curves with SAS. 1st ed. SAS Publishing.

22 Youden WJ. Index for rating diagnostic tests. Cancer. 1950;3(1):32.

23 Robin X, Turck N, Hainard A, Tiberti N, Lisacek F, Sanchez JC, et al. pROC: an opensource package for $\mathrm{R}$ and $\mathrm{S}+$ to analyze and compare ROC curves. BMC Bioinformatics. 2011;12:77.

24 Ogata N, Ikari Y, Nanasato M, Okutsu M, Kametani R, Abe M, et al. Safety margin of minimized contrast volume during percutaneous coronary intervention in patients with chronic kidney disease. Cardiovasc Interv Ther. 2014;29(3):209-15.
25 Laskey WK, Jenkins C, Selzer F, Marroquin OC, Wilensky RL, Glaser R, et al. NHLBI dynamic registry investigators: volume-to-creatinine clearance ratio: a pharmacokinetically based risk factor for prediction of early creatinine increase after percutaneous coronary intervention. J Am Coll Cardiol. 2007;50: 584-90.

26 Gurm HS, Dixon SR, Smith DE, Share D, Lalonde T, Greenbaum A, et al. BMC2 (blue cross blue shield of michigan cardiovascular consortium) registry: renal function-based contrast dosing to define safe limits of radiographic contrast media in patients undergoing percutaneous coronary interventions. J Am Coll Cardiol. 2011;58:907-14.

27 Taliercio CP, Vlietstra RE, Fisher LD, Burnett JC. Risks for renal dysfunction with cardiac angiography. Ann Intern Med. 1986;104(4): 501-4.

28 Gleeson TG, Bulugahapitiya S. Contrast-induced nephropathy. AJR Am J Roentgenol. 2004;183(6):1673-89.

29 Manske CL, Sprafka JM, Strony JT, Wang Y. Contrast nephropathy in azotemic diabetic patients undergoing coronary angiography. Am J Med. 1990;89(5):615-20.

30 CMS proposes IPPS changes for 2012 and beyond - www.hcpro.com [Internet]. Available from: http: //www.hcpro.com/HOM265196\%E2\%80\%936962/CMS-proposesIPPS-changes-for-2012-and-beyond.html; [cited 2020 Nov 16]

31 Hospital-Acquired Condition Reduction Program (HACRP) | CMS [Internet]. Available from: https://www.cms.gov/Medicare/ Medicare-Fee-for-Service-Payment/AcuteInpatientPPS/HAC-Reduction-Program [cited 2020 Nov 16]

32 Brown JR, Solomon RJ, Sarnak MJ, Mc Cullough PA, Splaine ME, Davies L, et al. Northern New England cardiovascular disease study group: reducing contrast-induced acute kidney injury using a regional multicenter quality improvement intervention. Circ Cardiovasc Qual Outcomes. 2014;7:693700 